\title{
MicroRNA-21 and its impact on signaling pathways in cervical cancer (Review)
}

\author{
YONG WANG $^{1}$, SHIYING ZHOU $^{1}$, KEFENG FAN $^{1}$ and CHEN JIANG $^{2}$ \\ Departments of ${ }^{1}$ Obstetrics and ${ }^{2}$ Gynecology, Jinan Maternity and Child Care Hospital, Jinan, Shandong 250002, P.R. China
}

Received April 1, 2018; Accepted November 29, 2018

DOI: $10.3892 / 01.2019 .10002$

\begin{abstract}
Oncogenic microRNA-21 (miR-21/miRNA-21) is a stable inhibitor of gene expression that is often upregulated in cervical cancer, a disease that affects the health of women and tends to transform and spread. Previous studies investigating miR-21 in biopsies and cells from cervical cancer patients have identified that miR-21 binds target mRNAs in signaling pathways or long non-coding RNAs (lncRNA). Furthermore, studies have elucidated the molecular mechanisms of two tumor necrosis factor $\alpha$ (TNF- $\alpha$ ) signaling pathways that promote cell proliferation and inhibit cell apoptosis. miR-21 inhibits the TNF receptor 1 (TNFR1) signaling pathway and activates the TNFR2 signaling pathway. Moreover, miR-21 enhances cervical cancer cell proliferation by influencing the protein kinase B/mammalian target of rapamycin and RAS p21 protein activator 1 signaling pathways. The present review discusses the evidence that miR-21 may impact cervical cancer through inhibiting apoptosis and enhancing proliferation, and may therefore be a target for clinical intervention.
\end{abstract}

\section{Contents}

1. Introduction

2. miR-21 expression and gene targeting in cervical cancer

3. miR-21 and the TNF- $\alpha /$ caspase-3/caspase- 8 signaling pathway

4. miR-21 and the AKT/mTOR signaling pathway

5. miR-21 and the RasA1 signaling pathway

6. miR-21 and other target molecules

7. Conclusions and perspectives

Correspondence to: Dr Chen Jiang, Department of Gynecology, Jinan Maternity and Child Care Hospital, 2 Jianguoxiao Jingsan Street, Shizhong, Jinan, Shandong 250002, P.R. China

E-mail: ztw777@126.com

Key words: microRNA-21, cervical cancer, signaling pathway, upregulation, tumor necrosis factor receptor

\section{Introduction}

Cervical cancer has a high incidence rate and poses a serious health risk to women. Nearly 50 million new patients with cervical cancer are recorded worldwide annually (1). At present, no effective treatment for patients with advanced cervical cancer exists. Therefore, identifying targets for diagnosis and treatment is essential $(2,3)$.

MicroRNAs (miRs/miRNAs) are small non-coding RNAs that regulate protein at the mRNA level; they are 18 to 22 nucleotides in length, and are ubiquitous in eukaryotes (4-6). A number of studies have reported the abnormal expression of miRNAs in cervical cancer, including increased expression of miR-21 and miR-218, and reduced expression of miR-34 and miR-145 (7-10). Alterations in the levels of these miRNAs have been demonstrated to influence the expression of certain steps in key signaling pathways, including caspase- 8 and caspase-3 in apoptosis, and phosphatase and tensin homolog (PTEN), an important tumor suppressor $(11,12)$.

miR-21 acts as an oncogene in cancer (13) by regulating a number of pathways involved in tumor development. In the present review, the signaling pathways regulated by miR-21 in cervical cancer were systematically summarized, including the TNF- $\alpha /$ caspase-3/caspase- $8, \mathrm{PI} 3 \mathrm{~K} / \mathrm{AKT} / \mathrm{mTOR}$ and RAS/MEK/ERK pathways. The impact of miR-21 on GAS5 lncRNA and TIMP3 was also discussed. miR-21 also regulates other pathways, including tropomyosin 1 , pro-apoptotic FAS ligand, B-cell translocation gene 2, Sprouty and F-box subfamily 1 (14). The present review provides an overall analysis of miR-21 and its impact in signaling pathways.

\section{2. miR-21 expression and gene targeting in cervical cancer}

The ability to influence cell proliferation by regulating miR-21 expression may prove to be a novel mechanism to prevent and treat cervical cancer. Upregulation of miR-21 expression has been demonstrated in numerous studies using patient tissue samples, whole blood and cervical cancer cell lines, including HeLa, SiHa and HT-3 cells (Table I) (7,11-13,15-18). These studies identified target genes, including von Hippel-Lindau tumor suppressor (VHL), tumor necrosis factor $\alpha$ (TNF- $\alpha$ ), tissue inhibitor of metalloproteinases 3 (TIMP3), growth arrest-specific 5 (GAS5), RAS p21 protein activator 1 (RASA1), programmed cell death 4 (PDCD4) and PTEN, as shown in Table I. The mechanism by which miR-21 
Table I. Targets of microRNA-21 in cervical cancer.

\begin{tabular}{|c|c|c|c|c|c|c|}
\hline First author & Year & Sample type & Target gene & Target region & Expression change & (Refs.) \\
\hline Cai et al & 2018 & $\begin{array}{l}\text { Cervical carcinoma cell } \\
\text { lines: } \mathrm{SiHa}, \mathrm{HeLa}, \mathrm{CaSki} \\
\text { C-4-1 and C-33-A }\end{array}$ & VHL & 3'-UTR & Downregulated & $(32)$ \\
\hline $\mathrm{Xu}$ et al & 2017 & HeLa Cells & TNF- $\alpha$ & Unknown & Upregulated & (11) \\
\hline Zhang et al & 2018 & $\begin{array}{l}\text { Cervical carcinoma tissues, } \\
\text { HeLa and SiHa cell lines }\end{array}$ & TIMP3 & 3'-UTR & Downregulated & (7) \\
\hline Wen et al & 2017 & Cervical cancer tissue & GAS5 & 3'-UTR & Downregulated & (13) \\
\hline Zhang et al & 2016 & $\begin{array}{l}\text { Whole-blood, HeLa and } \\
\text { HT- } 3 \text { cells }\end{array}$ & RasA1 & 3'-UTR & Downregulated & $(15)$ \\
\hline Chen et al & 2015 & HeLa cell & PDCD4 and PTEN & 3'-UTR & Upregulated & (12) \\
\hline Yao et al & 2009 & HeLa cells & PDCD4 & 3'-UTR & Downregulated & (18) \\
\hline
\end{tabular}

regulates TNF- $\alpha$ expression is unclear. miR-21 may regulate numerous signaling pathways via its target genes, including TNF- $\alpha /$ caspase-3/caspase-8, RASA1/mitogen-activated protein kinase kinase (MEK) and protein kinase B/mammalian target of rapamycin (AKT/mTOR) pathways. Certain target genes of miR-21 do not participate in these signaling pathways, including TIMP3 and GAS5 $(7,13)$.

\section{3. miR-21 and the TNF- $\alpha /$ caspase-3/caspase-8 signaling pathway}

Cell apoptosis is a complex, multistage process that involves numerous genes. Apoptosis can be induced by endoplasmic reticulum stress, the mitochondrial pathway and death receptor pathways. The expression and function of TNF- $\alpha$, caspase- 3 and caspase- 8 have been extensively studied in cancer apoptosis. These genes appear to serve important roles in disease pathogenesis and can be used as important prognostic markers. miR-21 has been shown to upregulate mRNA and protein expression levels of TNF- $\alpha$ through an unknown target in HeLa cells, thus impacting their proliferation (11). TNF- $\alpha$ has two receptors (TNFR1 and TNFR2) (19). As shown in Fig. 1, the cellular apoptosis program is activated when TNF- $\alpha$, which is upregulated by miR-21, binds and activates the TNFR1 receptor in HeLa cells (20). The proliferation capability of the cells is activated when TNF- $\alpha$ binds to TNFR2, upregulating nuclear factor $\kappa \mathrm{B}(\mathrm{NF}-\kappa \mathrm{B})$, and thus inhibiting caspase-3 and activating c-Jun N-terminal kinase (JNK) (11). Overexpression of miR-21 has been shown to activate the $\mathrm{NF}-\kappa \mathrm{B} / \mathrm{JNK}$ signaling pathway in certain studies (21-23).

\section{4. miR-21 and the AKT/mTOR signaling pathway}

Signaling through phosphoinositide3-kinase (PI3K) and its downstream targets, AKT and mTOR, serves a key role in differentiation, proliferation and cell survival (24). Tumor cell proliferation is increased by activating the PI3K/AKT/mTOR signaling pathways (Fig. 2). The tumor suppressor PTEN is a negative regulator of the PI3K/PTEN/AKT signaling pathways, and it targets AKT to regulate cellular functions, including cell growth, differentiation, proliferation and migration (25). PTEN is significantly upregulated in miR-21-knockout cells, indicating that miR-21 downregulates PTEN and reduces its negative regulation of the PI3K/PTEN/AKT signaling pathway (12).

PDCD4 is a tumor suppressor that inhibits translation initiation by binding to eukaryotic initiation factor 4A (eIF4A) (26). A number of experiments have shown that PDCD4 is an important target of miR-21 and can regulate the proliferation of cancer cells $(12,18)$. Downregulation of PDCD4 by miR-21 increases HeLa cell growth (18).

\section{5. miR-21 and the RasA1 signaling pathway}

KRAS is an oncogene that is frequently mutated in cancer, leading to constitutive activation of the RAS/MEK/extracellular signal-regulated kinase (ERK) signaling pathway, which promotes cell proliferation, anti-apoptosis signaling and malignant transformation (27). RasA1 is a member of the Ras-GTPase-activating family that inactivates KRAS. Luciferase activity assay in $293 \mathrm{~T}$ cells demonstrated the miR-21 targets the 3'-untranslated region of RasA1 mRNA (15). Furthermore, cell proliferation is increased by miR-21 targeting RasA1 and activating the RAS/MEK/ERK signaling pathway (Fig. 2) (28).

\section{6. miR-21 and other target molecules}

Long non-coding RNAs (lncRNAs) serve an important regulatory role in the biological activities of tumor cells. The accumulation of IncRNA GAS5, a tumor suppressor, in growth-arrested cells is regulated by the mTOR and 


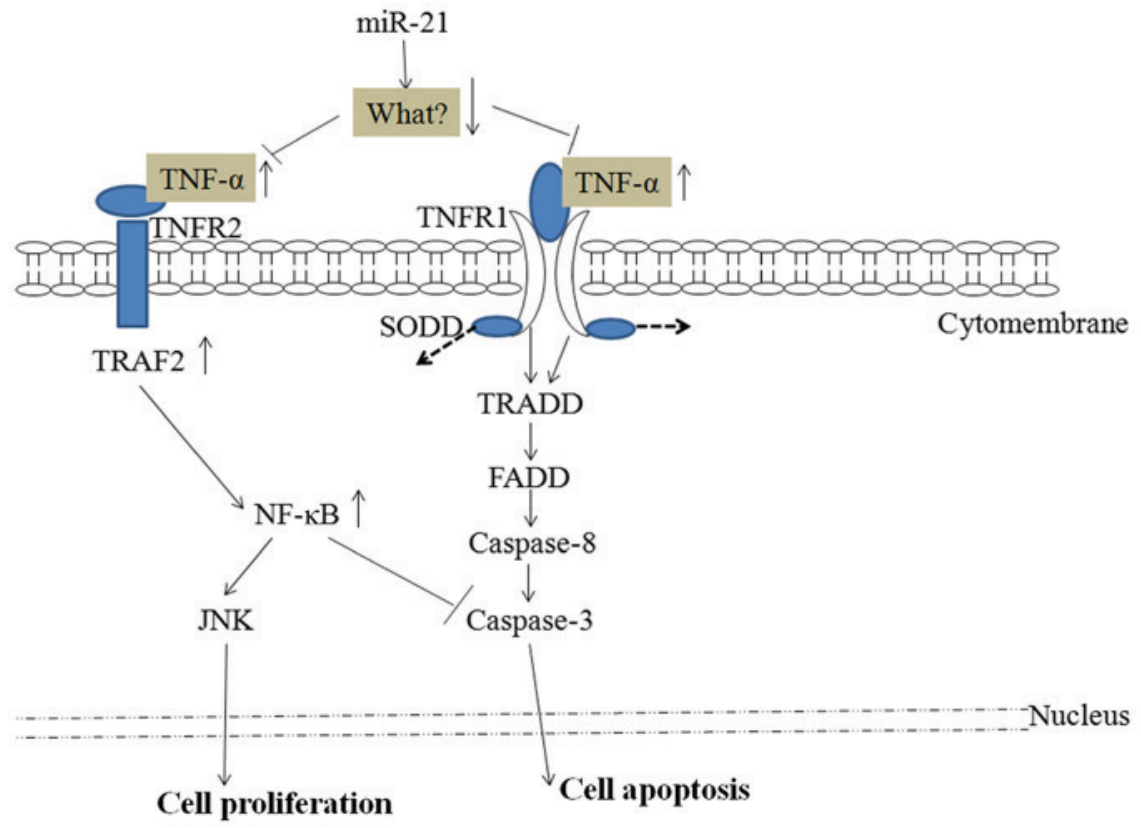

Figure 1. miR-21 and TNF- $\alpha$ signaling. miR-21, microRNA-21; TNF- $\alpha$, tumor necrosis factor $\alpha$; TNFR1/2, tumor necrosis factor receptors $1 / 2$; NF- $\kappa$ B, nuclear factor $\kappa \mathrm{B}$; JNK, c-Jun N-terminal kinase; TRAF2, TNF receptor-associated 2; SODD, silencer of death domain; TRADD, TNFRSF1A-associated via death domain; FADD, Fas-associated via death domain.

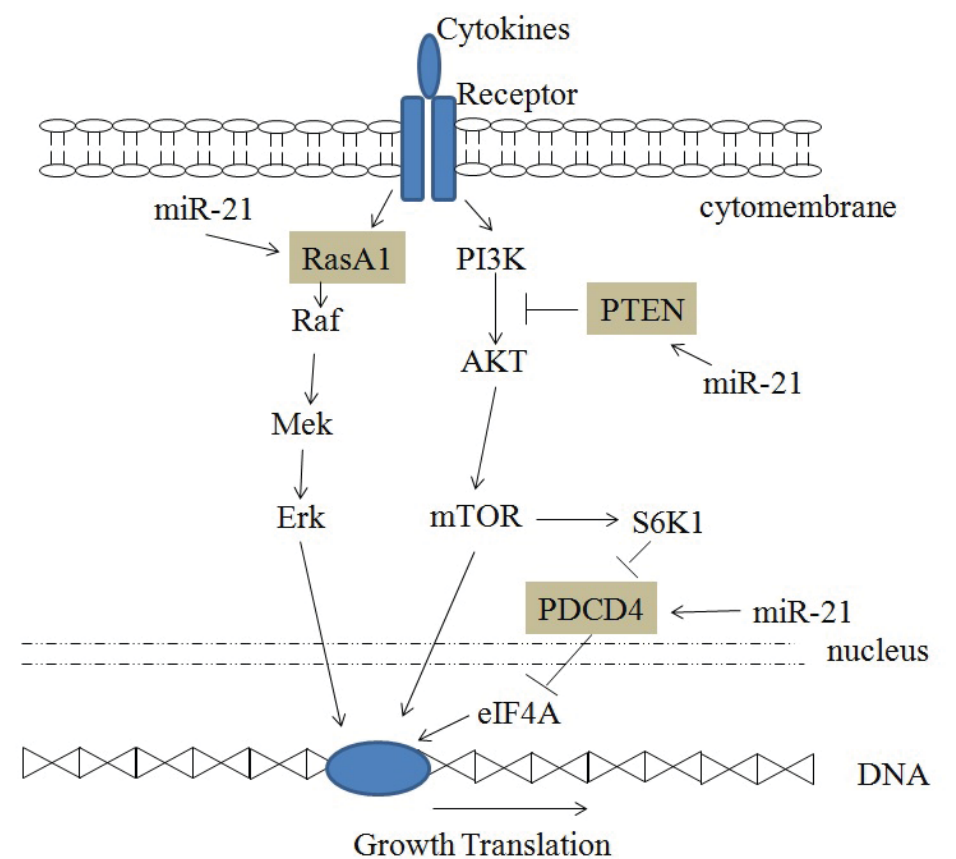

Figure 2. miR-21 and the AKT/mTOR and RasA1 signaling pathways. miR-21, microRNA-21; RasA1, RAS p21 protein activator 1; Raf, Raf proto-oncogene; Mek, mitogen-activated protein kinase kinase; Erk, extracellular signal-regulated kinase; PI3K, phosphoinositide 3-kinase; AKT, protein kinase B; mTOR, mammalian target of rapamycin; PTEN, phosphatase and tensin homolog; S6K1, ribosomal protein S6 kinase-1; PDCD4, programmed cell death 4; eIF4A, eukaryotic initiation factor 4A.

nonsense-mediated mRNA decay pathways (29). Wen et al (13) demonstrated that miR-21 directly targets GAS5 IncRNA, which can be used to diagnose the clinical stage of cervical cancer.

Deregulation of extracellular matrix homeostasis in cancer contributes to tumor growth and metastasis (30). This process is mediated by matrix metalloproteinases
(MMPs) and their inhibitors, including TIMP3, an independent promising biomarker in different cancer types. TIMP3 inhibits MMP activity to reduce the migration and invasion of cancer cells $(30,31)$. Zhang et al (7) found that miR-21 directly targets TIMP3 causing cervical cancer cells to become increasingly invasive and proliferative, and increasing their viability. 


\section{Conclusions and perspectives}

The present review provides insight into the effect of miR-21 on cervical cancer cells, thus supporting novel concepts for the diagnosis of the disease. As shown in Table I, miR-21 binds different target genes and regulates numerous signaling pathways, which alter cancer cells. miR-21 can be used as a biomarker of diagnosis and potentially as a therapeutic target.

The proliferation and apoptosis of cervical cancer cells requires the involvement and co-operation of numerous signaling molecules. The TNFR1/caspase signaling pathway via caspase-8/-3 can induce widespread cancer cell apoptosis upon binding to TNF- $\alpha$, which is regulated by miR-21 targeting of an as-yet-unknown intermediate (Fig. 1). Transcribed miR-21 can also upregulate cervical cancer cell proliferation via TNFR2 signaling by activating JNK and inhibiting caspase-3.

miR-21 can regulate other signaling pathways as shown in Fig. 2. Cervical cancer cell proliferation increases due to miR-21 binding and the inhibition of PTEN, thus inducing the PI3K/AKT/mTOR signaling pathway activity. Moreover, cell proliferation increases subsequent to miR-21 binding to RasA1, which inhibits the RAS/MEK/ERK signaling pathway. Furthermore, miR-21 can reduce the inhibition of eIF4A by PDCD4 and promote cell proliferation.

miR-21 has potential as a biomarker for the diagnosis and prognosis of cervical cancer, or as a treatment target in combination with other drugs to reduce metastasis. More research is essential to uncover the targets of miR-21 and its role in signaling pathways in cervical cancer, and to understand the mechanisms behind its activity.

\section{Acknowledgements}

Not applicable.

\section{Funding}

The authors were supported by the Technology Development Project Plan of Shandong Education Department (Shandong, China) (grant nos. J15LM63 and J14LM54).

\section{Availability of data and materials}

The datasets used or analyzed during the present study are available from the corresponding author on reasonable request.

\section{Authors' contributions}

YW was a major contributor in writing the manuscript. YW and CJ were responsible for the collection of the relevant literature. SZ and KF revised the manuscript critically for important intellectual content. All authors read and approved the final manuscript.

\section{Ethics approval and consent to participate}

Not applicable.

\section{Patient consent for publication}

Not applicable.

\section{Competing interests}

The authors declare that they have no competing interests.

\section{References}

1. Hoeben A, Polak J, Van De Voorde L, Hoebers F, Grabsch HI and de Vos-Geelen J: Cervical esophageal cancer: A gap in cancer knowledge. Ann Oncol 27: 1664-1674, 2016.

2. Buda A, Elisei F, Palazzi S, De Ponti E, Arosio M, Vecchione F, Dell'Anna T, Cuzzocrea M, Bussi B, Giuliani D, et al: Quality of care for cervical and endometrial cancer patients: The impact of different techniques of sentinel lymph node mapping on patient satisfaction. Ann Surg Oncol 23: 2975-2981, 2016.

3. Meier T and Kharofa J: Magnetic resonance imaging-guided high-dose rate brachytherapy for cervical cancer. Semin Roentgenol 51: 106-111, 2016.

4. Jiang XI, Luo Y,Zhao S, Chen Q, Jiang C, Dai Y, Chen Y and Cao Z: Clinical significance and expression of microRNA in diabetic patients with erectile dysfunction. Exp Ther Med 10: 213-218, 2015.

5. Jia W, Wu Y,Zhang Q, Gao GE, Zhang C and Xiang Y: Expression profile of circulating microRNAs as a promising fingerprint for cervical cancer diagnosis and monitoring. Mol Clin Oncol 3: 851-858, 2015.

6. Graziano A, Lo Monte G, Piva I, Caserta D, Karner M, Engl B and Marci R: Diagnostic findings in adenomyosis: A pictorial review on the major concerns. Eur Rev Med Pharmacol Sci 19: 1146-1154, 2015.

7. Zhang Z, Wang J, Wang X, Song W, Shi Y and Zhang L: MicroRNA-21 promotes proliferation, migration, and invasion of cervical cancer through targeting TIMP3. Arch Gynecol Obstet 297: 433-442, 2018.

8. Yang SF, Liu YF, Cheng CW, Yang WE, Lin WL, Ko JL and Wang PH: Impact of microRNA-34a and polymorphism of its target gene CA9 on susceptibility to uterine cervical cancer. Oncotarget 8: 77860-77871, 2017.

9. Zhou X, Yue Y, Wang R, Gong B and Duan Z: MicroRNA-145 inhibits tumorigenesis and invasion of cervical cancer stem cells. Int J Oncol 50: 853-862, 2017.

10. Jiang Z, Song Q, Zeng R, Li J, Li J, Lin X, Chen X, Zhang J and Zheng Y: MicroRNA-218 inhibits EMT, migration and invasion by targeting SFMBT1 and DCUN1D1 in cervical cancer. Oncotarget 7: 45622-45636, 2016.

11. Xu L, Xu Q, Li X and Zhang X: MicroRNA-21 regulates the proliferation and apoptosis of cervical cancer cells via tumor necrosis factor- $\alpha$. Mol Med Rep 16: 4659-4663, 2017.

12. Chen B, Chen X, Wu X, Wang X, Wang Y, Lin TY, Kurata J, Wu J, Vonderfecht S, Sun G, et al: Disruption of microRNA-21 by TALEN leads to diminished cell transformation and increased expression of cell-environment interaction genes. Cancer Lett 356: 506-516, 2015.

13. Wen Q, Liu Y, Lyu H, Xu X, Wu Q, Liu N, Yin Q, Li J and Sheng X: Long noncoding RNA GAS5, which acts as a tumor suppressor via microRNA 21, regulates cisplatin resistance expression in cervical cancer. Int J Gynecol Cancer 27: 1096-1108, 2017.

14. Shi J: Considering exosomal miR-21 as a biomarker for cancer. J Clin Med 5: pii: E42, 2016.

15. Zhang L, Zhan X, Yan D and Wang Z: Circulating MicroRNA-21 is involved in lymph node metastasis in cervical cancer by targeting RASA1. Int J Gynecol Cancer 26: 810-816, 2016.

16. Liu J, Sun H, Wang X, Yu Q, Li S, Yu X and Gong W: Increased exosomal microRNA-21 and microRNA-146a levels in the cervicovaginal lavage specimens of patients with cervical cancer. Int J Mol Sci 15: 758-773, 2014.

17. Gocze K, Gombos K, Juhasz K, Kovacs K, Kajtar B, Benczik M, Gocze P, Patczai B, Arany I and Ember I: Unique microRNA expression profiles in cervical cancer. Anticancer Res 33: 2561-2567, 2013.

18. Yao Q, Xu H, Zhang QQ, Zhou H and Qu LH: MicroRNA-21 promotes cell proliferation and down-regulates the expression of programmed cell death 4 (PDCD4) in HeLa cervical carcinoma cells. Biochem Biophys Res Commun 388: 539-542, 2009.

19. Qiu C, Hou G and Huang D: Molecular mechanism of TNF- $\alpha$ signal transduction. Chin J Biochemistry Mol Biol 23: 430-435, 2007.

20. Micheau O and Tschopp J: Induction of TNF receptor I-mediated apoptosis via two sequential signaling complexes. Cell 114: 181-190, 2003. 
21. Yang $\mathrm{Y}$ and Wang JK: The functional analysis of MicroRNA involved in NF-kappaB signaling. Eur Rev Med Pharmacol Sci 20: 1764-1774, 2016.

22. Gao Q, Xu L, Yang Q and Guan TJ: MicroRNA-21 contributes to high glucose-induced fibrosis in peritoneal mesothelia cells in rat models by activation of the Ras-MAPK signaling pathway via Sprouty-1. J Cell Physiol: Dec 4, 2018, (Epub ahead of print).

23. Zhang H, Wang Y, Lv Q, Gao J, Hu L and He Z: MicroRNA-21 overexpression promotes the neuroprotective efficacy of mesenchymal stem cells for treatment of intracerebral hemorrhage. Front Neurol 9: 931, 2018.

24. Zhang Z, Chen Q, Zhang J, Wang Y, Hu X, Yin S, He M, Guan S, Qin W, Xiao Q, et al: Associations of genetic polymorphisms in pTEN/AKT/mTOR signaling pathway genes with cancer risk: A meta-analysis in Asian population. Sci Rep 7: 17844, 2017.

25. Hu SL, Chang AC, Huang CC, Tsai CH, Lin CC and Tang CH: Myostatin promotes interleukin-1 $\beta$ expression in rheumatoid arthritis synovial fibroblasts through inhibition of miR-21-5p. Front Immunol 8: 1747, 2017.

26. Suzuki C, Garces RG, Edmonds KA, Hiller S, Hyberts SG, Marintchev A and Wagner G: PDCD4 inhibits translation initiation by binding to eIF4A using both its MA3 domains. Proc Natl Acad Sci USA 105: 3274-3279, 2008.
27. Gong B, Liu WW, Nie WJ, Li DF, Xie ZJ, Liu C, Liu YH, Mei P and Li ZJ: MiR-21/RASA1 axis affects malignancy of colon cancer cells via RAS pathways. World J Gastroenterol 21: 1488-1497, 2015.

28. Tidyman WE and Rauen KA: The RASopathies: Developmental syndromes of Ras/MAPK pathway dysregulation. Curr Opin Genet Dev 19: 230-236, 2009.

29. Mourtada-Maarabouni M and Williams GT: Growth arrest on inhibition of nonsense-mediated decay is mediated by noncoding RNA GAS5. Biomed Res Int 2013: 358015, 2013.

30. Martin del Campo SE, Latchana N, Levine KM, Grignol VP, Fairchild ET, Jaime-Ramirez AC, Dao TV, Karpa VI, Carson M, Ganju A, et al: MiR-21 enhances melanoma invasiveness via inhibition of tissue inhibitor of metalloproteinases 3 expression: In vivo effects of MiR-21 inhibitor. PLoS One 10: e0115919, 2015.

31. Nagao Y, Hisaoka M, Matsuyama A, Kanemitsu S, Hamada T, FukuyamaT,Nakano R,Uchiyama A, Kawamoto M, Yamaguchi K and Hashimoto H: Association of microRNA-21 expression with its targets, PDCD4 and TIMP3, in pancreatic ductal adenocarcinoma. Mod Pathol 25: 112-121, 2012.

32. Cai L, Wang W, Li X, Dong T, Zhang Q, Zhu B, Zhao H and Wu S: MicroRNA-21-5p induces the metastatic phenotype of human cervical carcinoma cells in vitro by targeting the von Hippel-Lindau tumor suppressor. Oncol Lett 15: 5213-5219, 2018. 